\title{
Glioblastoma multiforme: Effect of hypoxia and hypoxia inducible factors on therapeutic approaches (Review)
}

\author{
WEN-JUAN HUANG, WEI-WEI CHEN and XIA ZHANG \\ Department of Neurology, Xuzhou Central Hospital, Xuzhou, Jiangsu 221009, P.R. China
}

Received May 5, 2016; Accepted July 27, 2016

DOI: $10.3892 / \mathrm{ol} .2016 .4952$

\begin{abstract}
Central nervous system-based cancers have a much higher mortality rate with the 2016 estimates at 6.4 for incidence and 4.3 for deaths per 100,000 individuals. Grade IV astrocytomas, known as glioblastomas are highly aggressive and show a high proliferation index, diffused infiltration, angiogenesis, microvascular proliferation and pleomorphic vessels, resistance to apoptosis, and pseudopalisading necrosis. Extensive hypoxic regions in glioblastomas contribute to the highly malignant phenotype of these tumors. Hypoxic regions of glioblastoma exacerbate the prognosis and clinical outcomes of the patients as hypoxic tumor cells are resistant to chemo- and radiation therapy and are also protected by the malfunctional vasculature that developed due to hypoxia. Predominantly, hypoxia-inducible factor- $1 \alpha$, vascular endothelial growth factor (VEGF)/VEGF receptor, transforming growth factor- $\beta$, epidermal growth factor receptor and PI3 kinase/Akt signaling systems are involved in tumor progression and growth. Glioblastomas are predominantly glycolytic and hypoxia-induced factors are useful in the metabolic reprogramming of these tumors. Abnormal vessel formation is crucial in generating pseudopalisading necrosis regions that protect cancer stem cells residing in that region from therapeutic agents and this facilitates the cancer stem cell niche to expand and contribute to cell proliferation and tumor growth. Therapeutic approaches that target hypoxia-induced factors, such as use of the monoclonal antibody against VEGF, bevacizumab, have been useful only in stabilizing the disease but failed to increase overall survival. Hypoxia-activated TH-302, a nitroimidazole prodrug of cytotoxin bromo-isophosphoramide mustard, appears to be more attractive due to its better beneficial effects in glioblastoma patients. A better understanding of the hypoxia-mediated
\end{abstract}

Correspondence to: Dr Wen-Juan Huang, Department of Neurology, Xuzhou Central Hospital, 199 Jiefang South Road, Xuzhou, Jiangsu 221009, P.R. China

E-mail: ftmlb8556215@163.com

Key words: glioblastoma, astrocytoma, hypoxia, hypoxia-inducible factor-1 $\alpha$, vascular endothelial growth factor, pseudopalisading necrosis, cancer stem cells protection of glioblastoma cells is required for developing more effective therapeutics.

\section{Contents}

1. Introduction

2. Significance of hypoxia in development of glioblastoma

3. Hypoxia, growth factors and tumor angiogenesis

4. Significance of abnormal vessel formation in glioblastoma

5. Hypoxia and glioblastoma stem cells

6. Therapeutic implications

7. Conclusions

\section{Introduction}

Central nervous system (CNS)-based cancers account for approximately $1.4 \%$ of all cancers worldwide and account for proportionally more deaths, i.e., $2.7 \%$ among cancer-related mortalities (1). Estimates for incidence of CNS cancer in 2016 were $6.4 / 100,000$ individuals and for deaths this was 4.3/100,000 (2). Notably, of the many types of cells in CNS, most of the malignant tumors in brain originate from astrocytes. There are four distinct types of astrocytomas, classified on the basis of microscopic characteristics, grades (I-IV). Only the relatively rare grade I astrocytomas are curable while the other gliomas are incurable. Although grade III and IV gliomas are considered high-grade gliomas, only grade IV glioma is commonly known as glioblastoma multiforme. Glioblastomas show a high mitotic/proliferation index, diffused infiltration, angiogenesis, microvascular proliferation and pleomorphic vessel resistance to apoptosis, nuclear atypia, and pseudopalisading necrosis $(3,4)$. Gliomas progress with time from the time of diagnosis to aggressiveness and approximately $90 \%$ of astrocytoma present de novo as a glioblastoma. From the stage of glioblastoma occurrence, there is a poor survival rate, with a median of 16 months and survival rate of approximately $3 \%$ (5). It is extremely difficult to eliminate the glioblastoma even with total resection, as tumor cells persist microscopically, with tumor recurrence occurring in $90 \%$ of the patients at the original tumor location (6). The frequently seen extensive hypoxic regions in glioblastomas contribute to the highly malignant phenotype of these tumors, exacerbating the 


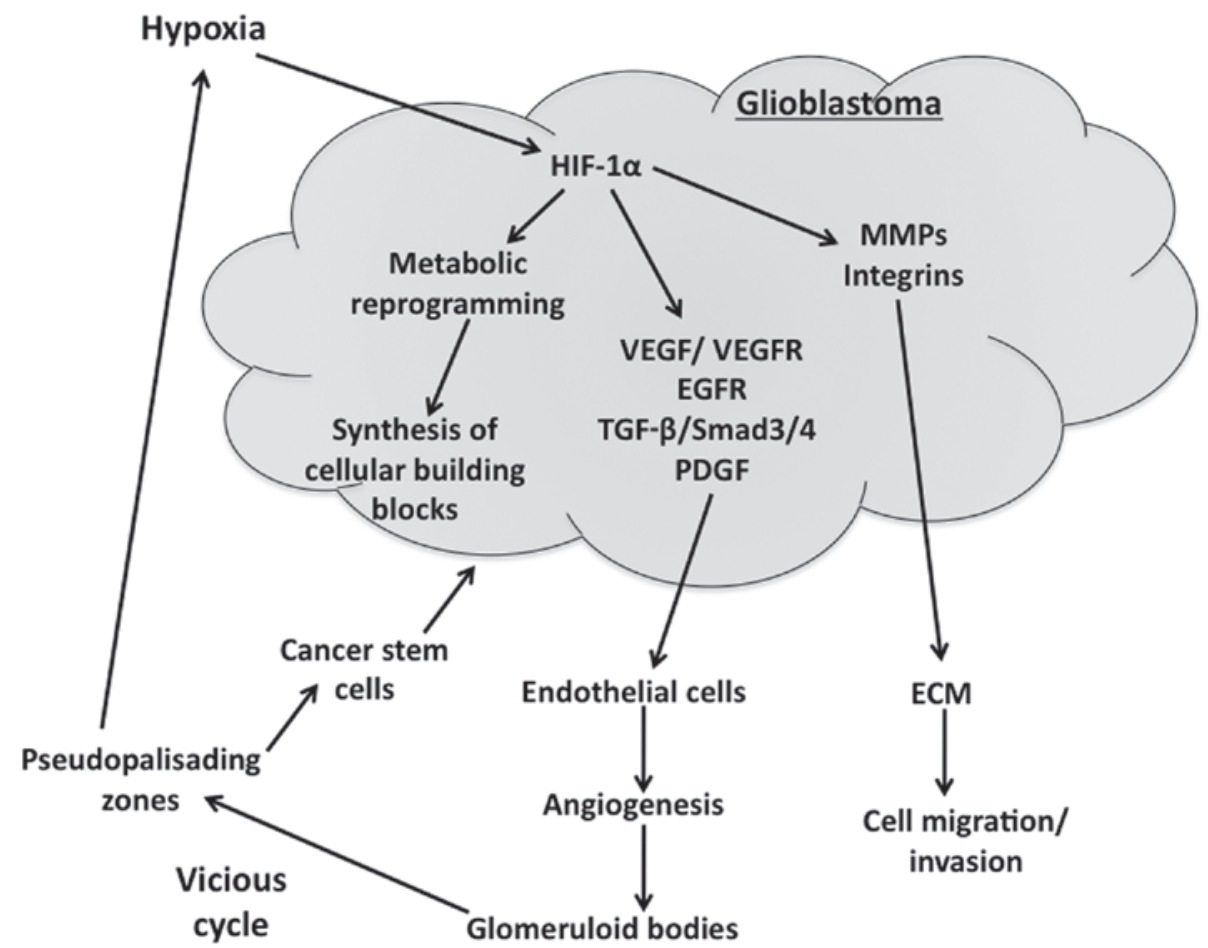

Figure 1. Hypoxia-driven events contribute to glioblastoma progression. Hypoxia stabilizes hypoxia-inducible factor-1 $\alpha$ (HIF-1 $\alpha$ ) in glioblastoma cells, thereby elevating the transcription of HIF-1 $\alpha$ targets. The major tumor-promoting targets of HIF-1 $\alpha$ are: Vascular endothelial growth factor (VEGF), VEGF receptor (VEGFR), epidermal growth factor receptor (EGFR), transforming growth factor- $\beta$ (TGF- $\beta$ ), plate-derived growth factor (PDGF), matrix metalloproteases (MMPs) and integrins. MMPs and integrins facilitate the migration of cancer cells through the extracellular matrix (ECM). VEGF, VEGFR and EGFR promote angiogenesis which leads to malfunctional glomeruloid vessel bodies. These malfunctional vessels lead to areas of pseudopalisading necrotic regions that are hypoxic and protect cancer stem cells, which further proliferate and lead to tumor expansion.

prognosis and clinical outcomes of the patients. Hypoxic tumor cells are more resistant to chemo- and radiation therapy $(7,8)$ and are protected by the vasculature that develops due to hypoxia-mediated molecular processes (3). Hypoxia also supports the survival of neural and glioma stem cells, which are drug resistant and possess tumorigenicity potential $(9,10)$. Considering the significance of hypoxia in the growth and aggressiveness of glioblastomas, targeting hypoxia potentially improves the outcomes in patients with this lethal cancer type.

\section{Significance of hypoxia in development of glioblastoma}

The pathognomonic feature of pseudopalisading necrosis, which is the area of hypercellularity surrounding necrotic regions, and vascular proliferation observed in glioblastoma tumors is a manifestation of hypoxia. These hypercellularity regions are highly hypoxic and represent tumor cells migrating away from vasoocclusive, distorted and degenerating blood vessels from the tumor center. Additionally, the cells have a high expression of hypoxia-inducible factor- $1 \alpha$ (HIF- $1 \alpha)$ and vascular endothelial growth factor (VEGF), which promote angiogenesis (11). A subset of growth factors including angiopoietins, fibroblast growth factors, chemokines and matrix metalloproteinases, play an important role in tumor angiogenesis (12). These new vessels are deformed, leaky and have gaps between endothelial cells, resulting in vascular stasis. Repeated cycle of events of angiogenesis, vascular collapse due to deformation and cancer cell migration, contribute to rapid tumor expansion in adjacent normal tissue and invasion (13). Inasmuch as hypoxia drives the progression and aggressiveness of glioblastoma tumors, a strategy for the treatment of this type of cancer has been developed by measuring tumor volume and the extent of intratumoral hypoxia, using fluoromisonidazole probe-based positron emission tomography, followed by appropriate targeting of hypoxic cells to improve the treatment outcome (14). As mentioned above, tumor stem cells residing in hypoxic pseudopalisading zones are protected from chemoradiation because of vascular stasis and depletion of molecular oxygen. In a prospective clinical trial testing bevacizumab and irinotecan in glioblastoma patients, it was observed that hypoxia-induced carbonic anhydrase (CA9) and HIF-2 $\alpha$ were major and significant predictors of treatment effectiveness and overall survival (15).

\section{Hypoxia, growth factors and tumor angiogenesis}

Hypoxic response is essentially mediated by HIF-1 $\alpha$, which is induced under conditions of low oxygen, in a nuclear factor- $\kappa \mathrm{B}$-dependent manner. HIF- $1 \alpha$ is a transcription factor that regulates the expression of approximately 60 genes involved in several cell pathways such as glycolysis, angiogenesis, invasion and epithelial-mesenchymal transition, which are critical for tumor growth and proliferation (Fig. 1). HIF-1 $\alpha$ likely mediates the hypoxic response, partly through the upregulation of glucose transporter 1 (Glut1) and glycolytic genes, thereby promoting anerobic glycolysis and metabolism to survive the unfavorable conditions encountered 
during hypoxia. Tumor initiating cancer stem cells undergo HIF-1 $\alpha$-mediated adaptation to hypoxia and thus have an elevated expression of various HIF-regulated genes (15). It has been shown that hypoxic area volume in the tumor is inversely proportional to the survival of the patients and thus hypoxic volume ratios greater than the median, survived only $\sim 4$ months as compared to $>12$ months in the patients with less than median hypoxic burden (14).

Multiple pro-angiogenic factors, which are under the regulation of HIF-1 $\alpha$ and other transcription factors, are present in glioblastoma and contribute to the formation of new vessels (Fig. 1). VEGF, a prominent pro-angiogenic factor and its receptor VEGF receptor 2 (VEGFR2), are highly expressed in glioblastomas and tumor vasculature $(17,18)$. In addition to angiogenesis, VEGF regulates vascular permeability and contributes to vasogenic edema. Most of the approaches developed for glioblastoma therapy that specifically address targeting angiogenesis, mainly focused on blocking VEGF signaling pathways (19). Primary glioblastomas also exhibit a high proportion of mutations and/or the overexpression of epidermal growth factor receptor (EGFR) gene. The EGFR gene codes for a $170-\mathrm{kDa}$ receptor, which is a tyrosine kinase receptor, and a glycosylated plasma membrane. Altered EGFR function often leads to oncogenesis in various cell types and contributes to glioblastoma initiation, cancer cell proliferation and growth, invasion, resistance to apoptosis, chemo- and radiotherapy and angiogenesis $(20,21)$. It has been observed that almost $40 \%$ of glioblastomas have amplified EGFR gene expression with $50 \%$ of them showing overexpression of the receptor protein (22). Even in less malignant astrocytomas and oligodendrogliomas, there are elevated EGFR mRNA levels, suggesting that other oncogenic events play a role in the amplification of the EGFR gene (23).

Another important growth factor is transforming growth factor- $\beta$ (TGF- $\beta$ ), whose signaling controls different cell functions such as proliferation, differentiation, and apoptosis and thus plays an important role in cancer progression, remodeling of the extracellular matrix and angiogenesis (24). The isoforms of TGF- $\beta$, TGF- $\beta 1 /-\beta 2$ and their complete cognate signaling machinery are known to be highly expressed in glioblastomas, and contribute to enhanced proliferation and invasion of tumor cells and tumor angiogenesis (25). TGF- $\beta 2$ has been shown to contribute to aberrant vascular gene expression via Smad 2/4 and Smad 3/4 signaling pathways in glioblastoma (26). Experimental studies indicated that plate-derived growth factor (PDGF)-B overexpression in murine neural and glial progenitor cells leads to the formation of malignant gliomas (27), and it is known that human glioblastomas show elevated expression of PDGF and PDGF-receptor. PDGF-B, derived from tumor cells is shown to enhance angiogenesis in endothelial cells via increased production of VEGF (28). The net effect on overall angiogenesis in glioblastoma tumors is dependent on the source and availability of PDGF-B and the counteracting effects of $\mathrm{Wnt} / \beta$-catenin signaling on angiogenesis (29).

\section{Significance of abnormal vessel formation in glioblastoma}

Glioblastomas are characterized by the presence of morphologically abnormal and dysfunctional vasculature, also known as glomeruloid bodies or vascular tufts (30). These glomeruloid bodies have multiple layers of endothelial cells, pericytes and smooth muscle cells and exhibit a thick basement membrane. Such morphology constitutes a typical diagnosis for glioblastoma, albeit not for low-grade gliomas (19). The origin of these vessels is not clear and controversial. Previous in vitro studies indicated that glioma stem-like cells can be induced to differentiate to an endothelial cell type and that glioblastoma stem-like cells contribute to vessel formation (Fig. 1) in experimental models (31,32). Although the blood-brain barrier, which consists of endothelial cells and pericytes astrocytes in normal brain vessels, is disrupted in glioblastoma, with a resultant increase in vascular permeability (33), this barrier appears to remain intact at the invasive front of the tumor and the normal cortex, which is invaded by migratory glioblastoma cells. Thus, therapeutic agents that target glioblastoma to cross the blood-brain barrier to attack the invasive glioblastoma cells. Of note, the malformed blood vessels in the glioblastoma tumors in turn contribute to increasing hypoxic conditions in the tumor, as these vessels are poorly perfused (34). As mentioned earlier, hypoxia is a potent inducer of tumor progression through metabolic reprogramming, resistance to cell death, immunosuppression, inflammation and epithelial-mesenchymal transition of cancer cells (13). The stabilized and thus elevated HIF- $1 \alpha$ in glioma cells causes an increase in the expression of VEGF and CXC chemokine ligand 12 , both of which promote angiogenesis through different mechanisms, eventually leading to the formation of the malformed vessels, thus leading to a vicious cycle that promotes glioblastoma tumor growth and aggressiveness (Fig. 1) (35).

\section{Hypoxia and glioblastoma stem cells}

Cancer stem cells are more potent in inducing tumors when injected into the brains of immunocompromised mice, as compared to non-stem cells from tumors. Cancer stem cells have a high level of resistance to radiation through activation of the DNA damage checkpoint and to chemoresistance $(36,37)$. An important function of the tumor vasculature is to provide a supply of glioblastoma stem-like cells (38), which are critical for the progression of tumor growth and resistance to chemo- and radiotherapy. Notably, hypoxia is shown to increase the expression of CD133, a stem cell marker, in brain tumors and this increase is probably mediated by the HIF family of transcription factors $(36,39)$. Of the HIF transcription factors, HIF-1 $\alpha$ promotes proliferation and survival of all cancer cells and is activated in normal neural progenitors, thus limiting its value, in terms of therapeutic targeting. HIF- $2 \alpha$, which is practically absent in non-glioma stem cells, is specifically elevated in glioblastoma stem cells, even under modest hypoxic conditions, thereby making HIF- $2 \alpha$ an interesting therapeutic target in glioblastomas (40). HIF-2 $\alpha$ regulates chromatin structure by activating proteins/processes that modify chromatin epigenetically, such as the histone methyltransferase and mixed lineage leukemia 1 (41). Other hypoxia-induced genes, that are expressed at elevated levels in glioblastoma stem cells, include Oct4, Glut1, SerpinB9, and VEGF. In addition to 
promoting angiogenesis by the glioma stem cells, hypoxia also increases other microenvironmental interactions by these cancer stem cells including the suppression of immune response. Hypoxia activates pSTAT signaling, thereby increasing the secretion of immunosuppressive cytokines such as CSF1 and CCL2, which are useful in inhibiting T-cell proliferation and macrophage phagocytosis, which in turn accelerate tumor progression (42).

Glioma stem cells show enhanced Notch signaling pathways $(43,44)$, which contribute to their radioresistance $(45)$. Thus, blocking Notch signaling in human glioblastoma stem cells with high doses of $\gamma$-secretase inhibitors reduces proliferation of these cancer stem cells and tumor formation, and increases differentiation (46). Other enhanced signaling pathways include PI3 kinase/Akt, Hedgehog and Stat3 (47-49).

\section{Therapeutic implications}

Inasmuch as hypoxia and hypoxia-induced factors promote the growth of glioblastomas, therapeutic measures addressing hypoxia have been developed. Thus, a monoclonal antibody against VEGF, bevacizumab, has been developed and approved as a second-line monotherapy of glioblastoma patients. However, bevacizumab therapy achieved improvement in progression-free survival but was not beneficial for overall survival (50). On the other hand, a subset of patients who positively responded to cediranib, a pan-VEGF receptor tyrosine kinase inhibitor, which increased blood perfusion, showed improved survival (51). A better understanding of the significance and pathophysiology of tumor vasculature and associated factors is needed to develop effective therapeutics that target these elements of glioblastomas.

An interesting direct approach using a prodrug is via a hypoxia-activated TH-302, which is a nitroimidazole prodrug of bromo-isophosphoramide mustard (Br-IPM), a cytotoxin. Under hypoxic conditions, TH-302 is converted by intracellular reductases to the alkylating agent Br-IPM, which functions as a DNA cross-linking agent damaging the hypoxic tumor cells, in which it is formed. Br-IPM may also diffuse into the extracellular matrix and the nearby normoxic cells, showing its cytotoxic effects in these cells as well. However, TH-302 per se is not effective under normoxic conditions and requires hypoxic conditions to show its cytotoxic effects (52). A recent study showed that TH-302 is well tolerated when administered after a 4-week period of postsurgical recovery, to glioblastoma patients, in combination with bevacizumab $(10 \mathrm{mg} / \mathrm{kg})$, with a promising clinical benefit rate of $62 \%$ (53).

A major advance in the treatment of glioblastoma during the last decade is the concomitant chemoradiotherapy with temozolomide. It has been shown that the median survival of patients receiving radiation therapy alone is 12.1 months, whereas a combination of radiation with temozolomide increased the median survival to $\leq 14.6$ months (54). One of 5 glioblastoma patients survived in the temozolomide-treated population, whereas essentially none of the patients without temozolomide survived to 3 years, during the follow-up. The survival promoting action of temozolomide is essentially due to the epigenetic modification of the methyl guanine methyl transferase (MGMT) promoter, in the glioblastoma cells, which leads to reduced or no expression of this enzyme and thus the inability to repair the guanine methylation induced by temozolomide. However, MGMT silencing by promoter modification is seen only in $40 \%$ of the glioblastoma cases. Therefore, the treatment benefit of temozolomide is not evident in the remaining $60 \%$ of the glioblastoma patients who harbor normal expression of the MGMT, which is capable of repairing the temozolomide-mediated guanine methylation (55).

Other agents that have been examined as therapeutic agents targeting HIFs are amphotericin B, an anti-fungal drug, which inhibits HIF-1 $\alpha$ transcription (56); and 2-methoxyestradiol, an inhibitor of HIF-1 $\alpha$, which has been tested in phase-I clinical trials with some success showing stable disease in $38 \%$ of the enrolled patients (57). Despite the significant leaps in our understanding of the molecular events that underlie the aggressiveness and progression of glioblastomas, much progress is needed for effective therapeutic development that goes much further than extending life by a short period of time.

\section{Conclusions}

Among the CNS cancer grade IV astrocytomas, glioblasomas are highly aggressive. The extensive hypoxic regions in glioblastomas contribute to the highly malignant phenotype of these tumors, exacerbating the prognosis of the patients. Hypoxic tumor cells are resistant to chemo- and radiation therapy and are also protected by the malfunctional vasculature that developed due to hypoxia. The abnormal and malfunctional vessels play a critical role in generating pseudopalisading necrotic regions that are hypoxic and protect cancer stem cells residing in that region from therapeutic agents, which facilitates cancer stem cell proliferation and tumor growth, thus causing a vicious cycle of tumor growth. Therapeutic approaches that target hypoxia-induced factors such as use of monoclonal antibody against VEGF, bevacizumab, have been useful only in stabilizing the disease but failed to increase the overall survival. Hypoxia-activated TH-302 appears to be more attractive due to its better beneficial effects in glioblastoma patients. A better understanding of the hypoxia mediated protection of the glioblastoma cells is needed in future studies in order to develop more effective therapeutics.

\section{References}

1. Filippini G: Epidemiology of primary central nervous system tumors. Handb Clin Neurol 104: 3-22, 2012.

2. Howlader N, Noone A, Krapcho M, Miller D, Bishop K, Altekruse S, Kosary C, Yu M, Ruhl J, Tatalovich Z, et al (eds): Seer cancer statistics review, 1975-2013. National Cancer Institute, Bethesda, MD. http://seer.Cancer.Gov/csr/1975_2013/. Accessed April 15, 2016.

3. Louis DN, Ohgaki H, Wiestler OD, Cavenee WK, Burger PC, Jouvet A, Scheithauer BW and Kleihues P: The 2007 WHO classification of tumours of the central nervous system. Acta Neuropathol 114: 97-109, 2007.

4. Furnari FB, Fenton T, Bachoo RM, Mukasa A, Stommel JM, Stegh A, Hahn WC, Ligon KL, Louis DN, Brennan C, et al: Malignant astrocytic glioma: Genetics, biology, and paths to treatment. Genes Dev 21: 2683-2710, 2007.

5. Ostrom QT, Gittleman H, Liao P, Rouse C, Chen Y, Dowling J, Wolinsky Y, Kruchko C and Barnholtz-Sloan J: CBTRUS statistical report: Primary brain and central nervous system tumors diagnosed in the United States in 2007-2011. Neuro-oncol 16 (Suppl 4): iv1-iv63, 2014. 
6. Hou LC, Veeravagu A, Hsu AR and Tse VC: Recurrent glioblastoma multiforme: A review of natural history and management options. Neurosurg Focus 20: E5, 2006.

7. Rong Y, Durden DL, Van MeirEG and Brat DJ: 'Pseudopalisading' necrosis in glioblastoma: A familiar morphologic feature that links vascular pathology, hypoxia, and angiogenesis. J Neuropathol Exp Neurol 65: 529-539, 2006.

8. Hsieh CH, Shyu WC, Chiang CY, Kuo JW, Shen WC and Liu RS: NADPH oxidase subunit 4-mediated reactive oxygen species contribute to cycling hypoxia-promoted tumor progression in glioblastoma multiforme. PLoS One 6: e23945, 2011.

9. Singh SK, Hawkins C, Clarke ID, Squire JA, Bayani J, Hide T, Henkelman RM, Cusimano MD and Dirks PB: Identification of human brain tumour initiating cells. Nature 432: 396-401, 2004.

10. Bao S, Wu Q, McLendon RE, Hao Y, Shi Q, Hjelmeland AB, Dewhirst MW, Bigner DD and Rich JN: Glioma stem cells promote radioresistance by preferential activation of the DNA damage response. Nature 444: 756-760, 2006.

11. Brat DJ, Castellano-Sanchez AA, Hunter SB, Pecot M, Cohen C, Hammond EH, Devi SN, Kaur B and Van Meir EG: Pseudopalisades in glioblastoma are hypoxic, express extracellular matrix proteases, and are formed by an actively migrating cell population. Cancer Res 64: 920-927, 2004

12. Jain RK, di Tomaso E, Duda DG, Loeffler JS, Sorensen AG and Batchelor TT: Angiogenesis in brain tumours. Nat Rev Neurosci 8: 610-622, 2007.

13. Jain RK: Normalizing tumor microenvironment to treat cancer: Bench to bedside to biomarkers. J Clin Oncol 31: 2205-2218, 2013.

14. Spence AM, Muzi M, Swanson KR, O'Sullivan F, Rockhill JK, Rajendran JG, Adamsen TC, Link JM, Swanson PE, Yagle KJ, et al: Regional hypoxia in glioblastoma multiforme quantified with [18F]fluoromisonidazole positron emission tomography before radiotherapy: Correlation with time to progression and survival. Clin Cancer Res 14: 2623-2630, 2008.

15. Sathornsumetee S, Cao Y, Marcello JE, Herndon JE II, McLendon RE, Desjardins A, Friedman HS, Dewhirst MW, Vredenburgh JJ and Rich JN: Tumor angiogenic and hypoxic profiles predict radiographic response and survival in malignant astrocytoma patients treated with bevacizumab and irinotecan. J Clin Oncol 26: 271-278, 2008.

16. Mannino $\mathrm{M}$ and Chalmers AJ: Radioresistance of glioma stem cells: Intrinsic characteristic or property of the "microenvironment-stem cell unit'? Mol Oncol 5: 374-386, 2011.

17. Plate KH, Breier G, Weich HA and Risau W: Vascular endothelial growth factor is a potential tumour angiogenesis factor in human gliomas in vivo. Nature 359: 845-848, 1992.

18. Olsson AK, Dimberg A, Kreuger J and Claesson-Welsh L: VEGF receptor signalling - in control of vascular function. Nat Rev Mol Cell Biol 7: 359-371, 2006

19. Dimberg A: The glioblastoma vasculature as a target for cancer therapy. Biochem Soc Trans 42: 1647-1652, 2014

20. Karpel-Massler G, Schmidt U, Unterberg A and Halatsch ME: Therapeutic inhibition of the epidermal growth factor receptor in high-grade gliomas: Where do we stand? Mol Cancer Res 7: 1000-1012, 2009.

21. Squatrito M and Holland EC: DNA damage response and growth factor signaling pathways in gliomagenesis and therapeutic resistance. Cancer Res 71: 5945-5949, 2011

22. Riddick $\mathrm{G}$ and Fine HA: Integration and analysis of genome-scale data from gliomas. Nat Rev Neurol 7: 439-450, 2011.

23. Reifenberger J, Reifenberger G, Ichimura K, Schmidt EE, Wechsler W and Collins VP: Epidermal growth factor receptor expression in oligodendroglial tumors. Am J Pathol 149: 29-35, 1996.

24. Massagué J: TGFbeta in Cancer. Cell 134: 215-230, 2008

25. Bruna A, Darken RS, Rojo F, Ocaña A, Peñuelas S, Arias A, Paris R, Tortosa A, Mora J, Baselga J, et al: High TGFbeta-Smad activity confers poor prognosis in glioma patients and promotes cell proliferation depending on the methylation of the PDGF-B gene. Cancer Cell 11: 147-160, 2007.

26. Dieterich LC, Mellberg S, Langenkamp E, Zhang L, Zieba A, Salomäki H, Teichert M, Huang $\mathrm{H}$, Edqvist PH, Kraus T, et al: Transcriptional profiling of human glioblastoma vessels indicates a key role of VEGF-A and TGF32 in vascular abnormalization. J Pathol 228: 378-390, 2012.

27. Shih AH and Holland EC: Platelet-derived growth factor (PDGF) and glial tumorigenesis. Cancer Lett 232: 139-147, 2006.
28. Guo P, Hu B, Gu W, Xu L, Wang D, Huang HJ, Cavenee WK and Cheng SY: Platelet-derived growth factor-B enhances glioma angiogenesis by stimulating vascular endothelial growth factor expression in tumor endothelia and by promoting pericyte recruitment. Am J Pathol 162: 1083-1093, 2003.

29. Lindblom P, Gerhardt H, Liebner S, Abramsson A, Enge M, Hellstrom M, Backstrom G, Fredriksson S, Landegren U, Nystrom HC, et al: Endothelial PDGF-B retention is required for proper investment of pericytes in the microvessel wall. Genes Dev 17: 1835-1840, 2003.

30. Kanu OO, Mehta A, Di C, Lin N, Bortoff K, Bigner DD, Yan H and Adamson DC: Glioblastoma multiforme: A review of therapeutic targets. Expert Opin Ther Targets 13: 701-718, 2009.

31. Ricci-Vitiani L, Pallini R, Biffoni M, Todaro M, Invernici G, Cenci T, Maira G, Parati EA, Stassi G, Larocca LM, et al: Tumour vascularization via endothelial differentiation of glioblastoma stem-like cells. Nature 468: 824-828, 2010.

32. Soda Y, Marumoto T, Friedmann-Morvinski D, Soda M, Liu F, Michiue H, Pastorino S, Yang M, Hoffman RM, Kesari S, et al: Transdifferentiation of glioblastoma cells into vascular endothelial cells. Proc Natl Acad Sci USA 108: 4274-4280, 2011.

33. Wolburg H, Noell S, Fallier-Becker P, Mack AF and Wolburg-Buchholz K: The disturbed blood-brain barrier in human glioblastoma. Mol Aspects Med 33: 579-589, 2012

34. Gmeiner M, Sonnberger M, Wurm G and Weis S: Glioblastoma with the appearance of arteriovenous malformation: Pitfalls in diagnosis. Clin Neurol Neurosurg 115: 501-506, 2013.

35. Würth R, Bajetto A, Harrison JK, Barbieri F and Florio T: CXCL12 modulation of CXCR4 and CXCR7 activity in human glioblastoma stem-like cells and regulation of the tumor microenvironment. Front Cell Neurosci 8: 144, 2014.

36. Blazek ER, Foutch JL and Maki G: Daoy medulloblastoma cells that express CD133 are radioresistant relative to CD133- cells, and the CD133+ sector is enlarged by hypoxia. Int J Radiat Oncol Biol Phys 67: 1-5, 2007

37. Todaro M, Alea MP, Di Stefano AB, Cammareri P, Vermeulen L, Iovino F, Tripodo C, Russo A, Gulotta G, Medema JP, et al: Colon cancer stem cells dictate tumor growth and resist cell death by production of interleukin-4. Cell Stem Cell 1: 389-402, 2007.

38. Calabrese C, Poppleton H, Kocak M, Hogg TL, Fuller C, Hamner B, Oh EY, Gaber MW, Finklestein D, Allen M, et al: A perivascular niche for brain tumor stem cells. Cancer Cell 11: 69-82, 2007.

39. Platet N, Liu SY, Atifi ME, Oliver L, Vallette FM, Berger F and Wion D: Influence of oxygen tension on CD133 phenotype in human glioma cell cultures. Cancer Lett 258: 286-290, 2007.

40. Li Z, Bao S, Wu Q, Wang H, Eyler C, Sathornsumetee S, Shi Q, Cao Y, Lathia J, McLendon RE, et al: Hypoxia-inducible factors regulate tumorigenic capacity of glioma stem cells. Cancer Cell 15: 501-513, 2009.

41. Heddleston JM, Wu Q, Rivera M, Minhas S, Lathia JD, Sloan AE, Iliopoulos O, Hjelmeland AB and Rich JN: Hypoxia-induced mixed-lineage leukemia 1 regulates glioma stem cell tumorigenic potential. Cell Death Differ 19: 428-439, 2012.

42. Wei J, Wu A, Kong LY, Wang Y, Fuller G, Fokt I, Melillo G, Priebe $\mathrm{W}$ and Heimberger AB: Hypoxia potentiates gliomamediated immunosuppression. PLoS One 6: e16195, 2011.

43. Kanamori M, Kawaguchi T, Nigro JM, Feuerstein BG, Berger MS, Miele L and Pieper RO: Contribution of Notch signaling activation to human glioblastoma multiforme. J Neurosurg 106: 417-427, 2007.

44. Lino MM, Merlo A and Boulay JL: Notch signaling in glioblastoma: A developmental drug target? BMC Med 8: 72, 2010.

45. Wang J, Wakeman TP, Lathia JD, Hjelmeland AB, Wang XF, White RR, Rich JN and Sullenger BA: Notch promotes radioresistance of glioma stem cells. Stem Cells 28: 17-28, 2010.

46. Fan X, Khaki L, Zhu TS, Soules ME, Talsma CE, Gul N, Koh C, Zhang J, Li YM, Maciaczyk J, et al: NOTCH pathway blockade depletes CD133-positive glioblastoma cells and inhibits growth of tumor neurospheres and xenografts. Stem Cells 28: 5-16, 2010.

47. Wei Y, Jiang Y, Zou F, Liu Y, Wang S, Xu N, Xu W, Cui C, Xing Y, Liu Y, Cao B, Liu C, Wu G, Ao H, Zhang X and Jiang : Activation of PI3K/Akt pathway by CD133-p85 interaction promotes tumorigenic capacity of glioma stem cells. Proc Natl Acad Sci USA 110: 6829-6834, 2013.

48. Morgenroth A, Vogg AT, Ermert K, Zlatopolskiy B and Mottaghy FM: Hedgehog signaling sensitizes glioma stem cells to endogenous nano-irradiation. Oncotarget 5: 5483-5493, 2014. 
49. Liu M, Inoue K, Leng T, Guo S and Xiong ZG: TRPM7 channels regulate glioma stem cell through STAT3 and Notch signaling pathways. Cell Signal 26: 2773-2781, 2014

50. Keunen O, Johansson M, Oudin A, Sanzey M, Rahim SA, Fack F, Thorsen F, Taxt T, Bartos M, Jirik R, et al: Anti-VEGF treatment reduces blood supply and increases tumor cell invasion in glioblastoma. Proc Natl Acad Sci USA 108: 3749-3754, 2011.

51. Sorensen AG, Emblem KE, Polaskova P, Jennings D, Kim $\mathrm{H}$ Ancukiewicz M, Wang M, Wen PY, Ivy P, Batchelor TT, et al: Increased survival of glioblastoma patients who respond to antiangiogenic therapy with elevated blood perfusion. Cancer Res 72: 402-407, 2012.

52. Meng F, Evans JW, Bhupathi D, Banica M, Lan L, Lorente G, Duan JX, Cai X, Mowday AM, Guise CP, et al: Molecular and cellular pharmacology of the hypoxia-activated prodrug TH-302. Mol Cancer Ther 11: 740-751, 2012.

53. Cavazos DA and Brenner AJ: Hypoxia in astrocytic tumors and implications for therapy. Neurobiol Dis 85: 227-233, 2016.

54. Stupp R, Mason WP, van den Bent MJ, Weller M, Fisher B, Taphoorn MJ, Belanger K, Brandes AA, Marosi C, Bogdahn U, et al; European Organisation for Research and Treatment of Cancer Brain Tumor and Radiotherapy Groups; National Cancer Institute of Canada Clinical Trials Group: Radiotherapy plus concomitant and adjuvant temozolomide for glioblastoma. $\mathrm{N}$ Engl J Med 352: 987-996, 2005.
55. Hegi ME, Diserens AC, Gorlia T, Hamou MF, de Tribolet N, Weller M, Kros JM, Hainfellner JA, Mason W, Mariani L, et al: MGMT gene silencing and benefit from temozolomide in glioblastoma. N Engl J Med 352: 997-1003, 2005.

56. Yeo EJ, Ryu JH, Cho YS, Chun YS, Huang LE, Kim MS and Park JW: Amphotericin B blunts erythropoietin response to hypoxia by reinforcing FIH-mediated repression of HIF-1. Blood 107: 916-923, 2006.

57. Kirkpatrick J, Desjardins A, Quinn J, Rich J, Vredenburgh J, Sathornsumetee S, Gururangan S, Sidor C, Friedman H, Reardon D: Phase ii open-label, safety, pharmacokinetic and efficacy study of 2-methoxyestradiol nanocrystal colloidal dispersion administered orally to patients with recurrent glioblastoma multiforme. J Clin Oncol (ASCO Annual Meeting abs.) 25: 2065,2007 